\title{
Optical coherence tomography - a useful examination in the fight against multiple sclerosis
}

\author{
Anabella-Cristiana Cioabla, Cecilia Adumitresi, Ninela Radulescu, Ileana Ion
}

"Ovidius" University, Faculty of Medicine, Constanta, Romania

\begin{abstract}
Multiple sclerosis is a demyelinating disease that affects the young adult and produces major disability. The retina of the patients with multiple sclerosis displays inflammatory and neurodegenerative findings. Optical coherence tomography is a modern, method used to explore the retina.

In the last decade this technique has been applied in several areas in neurology. We present the case of a 27 -yearold patient with multiple sclerosis. The initial manifestation was optic neuritis. RNFL thinning resulted from OCT examination reflects axonal degeneration.
\end{abstract}

Keywords: multiple sclerosis, optical coherence tomography

\begin{abstract}
Abbreviations (in alphabetical order)
MRI - Magnetic Resonance Imaging

MS - Multiple sclerosis

OCT - Optical coherence tompgraphy

$\mathrm{ON}$ - optic neuritis

RNFL - retinal nerve fiber layer
\end{abstract}

\section{INTRODUCTION}

Multiple sclerosis (MS) is a chronic, demyelinating disease that involves the central nervous system. It is the most frequent neurological disease affecting the young adult and producing major disability (1).

The physiopathology of MS is characterized by demyelination. Axonal and neural degeneration, are probably the processes responsable for the persistent disability in the disease (2).

Clinical features depend on the localization of the demielynating lesions. Frequent clinical manifestations are: motor deficit, paresthesias, dysarthria, loss of sight, diplopia, tremor, ataxia, deep sensation impairment, bladder dysfunction (3).

The diagnosis is based on clinical features and imaging studies MRI. There is no curative treatment. The available therapies are meant to increase duration of time between relapses and to delay major disability.

Optic neuritis (ON) is one of the most common manifestations in multiple sclerosis (4). Is the initial manifestation in $25 \%$ of the cases.

The symptoms are: sudden vision loss and pain within the orbit. The pain is increased by eye movements It starts with blurry vision and it can evolve to complete vision loss or, in rare cases complete loss of light perception..

Physical exam can reveal decreased visual acuity and central scotoma (3).

Optical coherence tomography (OCT) is a non invasive, non- contact method used to explore the retina. The method is using the principle of interferometry in low coherence. OCT allows obtaining longitudinal sections of the retina, the axial resolution is about $10 \mu \mathrm{m}$ and the frontal resolution is aprox. $60 \mu \mathrm{m}(5)$. 
In the last decade this technique has been applied in several areas in neurology, demonstrating its potential role as a fundamental tool in the study of neurodegenerative diseases, such as MS, Parkinson's disease, or Alzeheimer's disease (6).

The retina is a unique structure in the central nervous system, containing axons, glial cells and no myelin, this why it is the ideal structure to explore in order to follow uo the neurodegenerative processes (2).

In MS patients, the retina displays inflammatory and neurodegenerative findings, such as perivascular inflammatory infiltrates and atrophy of the inner retinal layers.

Both severity and disease progression can be correlated to retinal nerve fiber layer (RNFL) thickness (6).

Compared to healthy subjects, MS patients present with a higher reduction and more affected sectors of the peripapillary RNFL thickness, and this reduction seems to be even greater in untreated patients. More recently, the peripapillary RNFL thickness was pointed out as a good predictor of the likelihood of disability worsening in MS patients over time (6).

\section{CASE PRESENTATION}

A 27 year old male patient presentend to the ophthalmology department with a 2 years history of right eye blurring of vision, accompanied by pain on eye movement. The symptoms appeared suddenly and slightly improved a couple weeks after onset.

There was no past medical history of note and he was not on regular medications. From family history: the mother suffers from autoimmune thyroiditis and, he has one sister diagnosed with systemic lupus erythematosus.

On examination, visual acuity in the right eye is 0.4 without correction and does not improve with correction. The left eye visual acuity is 1 without correction. Fundus examination showed pale right optic nerve head.

OCT examination was performed with Zeiss Stratus OCT. The investigation revealed decreased RNFL thickness $(82.35 \mu \mathrm{m})$ in the temporal section of the right eye. The values considered to be normal are between 87.2 and $109 \mu \mathrm{m}(98.1+/-10.9 \mu \mathrm{m})$.

The ophthalmologist based on the results of the investigations suspects the diagnosis of right optic

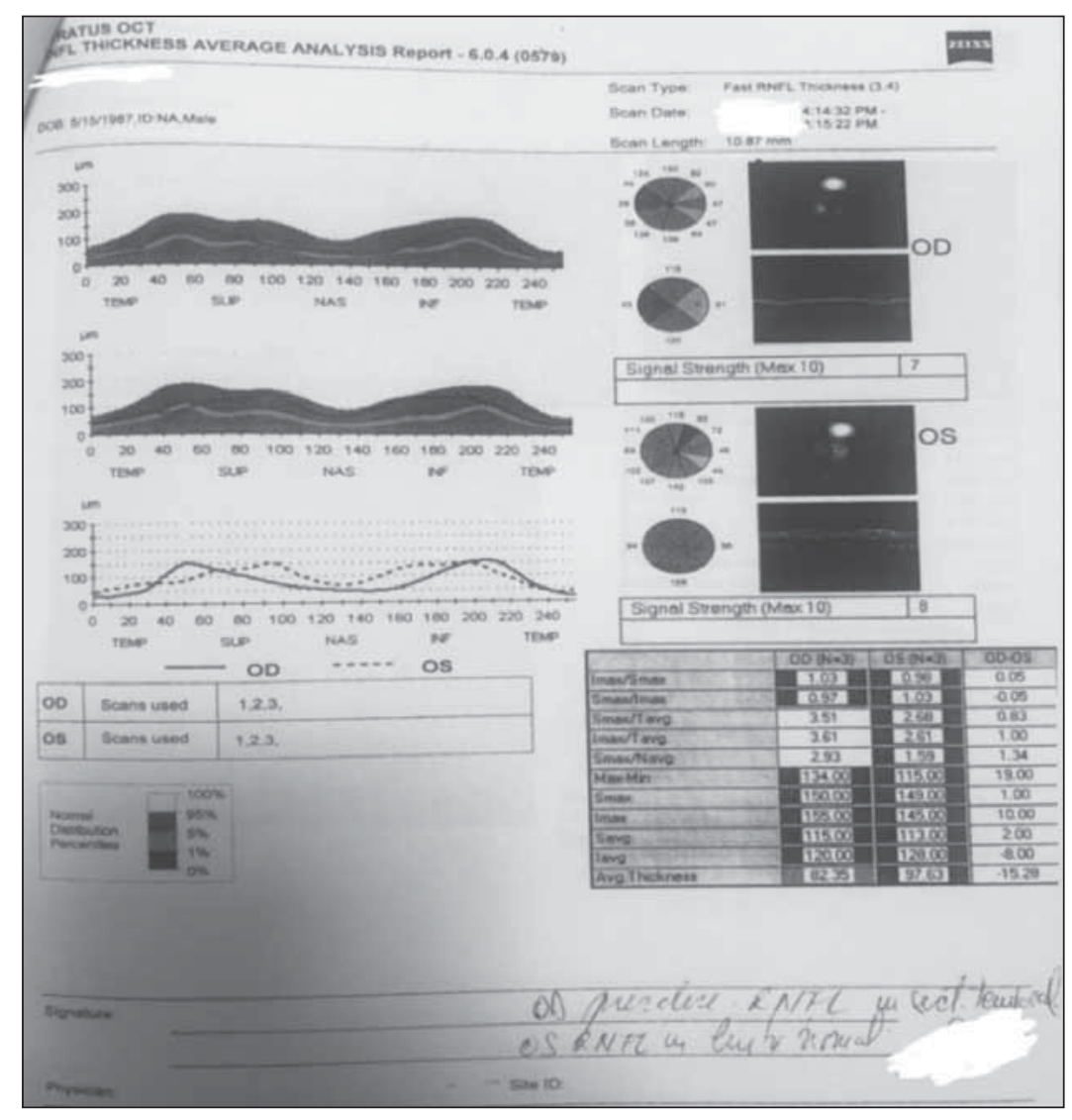

FIGURE 1. Optical coherence tomography generated by Zeiss Stratus OCT. Average RNFL thickness is indicated in the bottom row of the table. The clockface sector and the quadrant measures RNFL. In the temporal quadrant of the right eye there is a reduction in RNFL thickness (the dark shade). 
neuritis and recommends a neurologic examination to the patient.

Objective neurological examination revealed oriented, cooperative patient. Visual acuity examined from a $5 \mathrm{~m}$ distance was 0.4 in the right eye and 1 in the left eye. The rest of the cranial nerves resulted normal. No abnormal signs were detected during pyramidal, extrapyramidal, cerrebelar or sensory testing. The gait was normal.

MRI of the brain revealed multiple lesions with increased FLAIR and T2 signal, nonenhancing, in the periventricular white matter, and, three contrast enhancing (active) lesions.

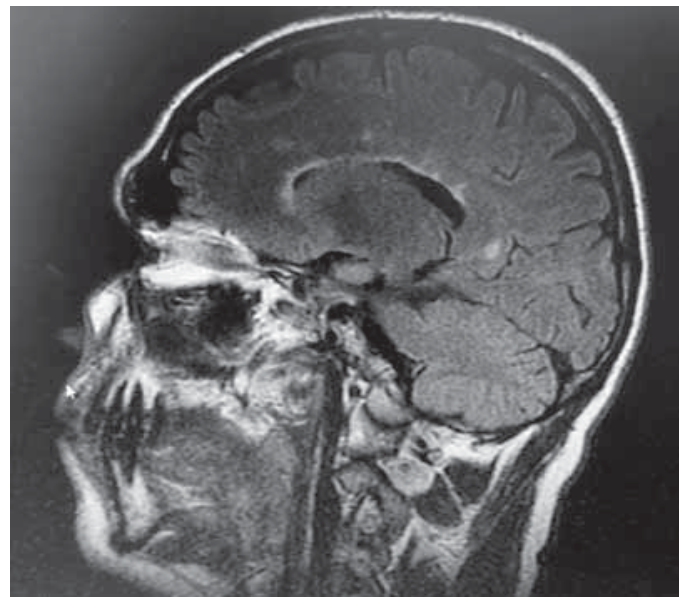

FIGURE 2. Cerebral MRI. Sagital section indicating hyperintense T2 periventricular white matter lesions.

Contrast cervical spine MRI revealed two nonenhancing hyperintense $\mathrm{T} 2$ lesions extending from $\mathrm{C} 2$ to $\mathrm{C} 5$.

For differential diagnosis the following laboratory tests were performed: Aquaporin 4 antibodies, ds DNA antibodies, pANCA, cANCA, Borreliosis testing, HIV testing, $\mathrm{B}$ and $\mathrm{C}$ viral testing- negative. Vitamin B12 was normal. There is increased value for TPO antibodies - $294.0 \mathrm{UI} / \mathrm{ml}$ (normal < $5.6 \mathrm{UI} / \mathrm{ml})$.

Endocrinological examination and specific thyroid laboratory tests established the diagnosis of autoimmune thyroiditis with normal thyroid function.
Based on the clinical features, MRI findings and laboratory testing, MS diagnosis was established. The patient was recommended to attend the Multiple Sclerosis National Treatment Programme.

$\mathrm{He}$ is on cronic treatment with beta interferon, $30 \mathrm{mcg} /$ week. Of course, in case of relapse he will be treated with methyilprednisolone .

\section{DISCUSSION}

We report a case of MS in a young male patient with $\mathrm{ON}$ as a first clinical manifestation of the disease. Decreased RNFL thickness as provided by OCT indicates involvement of the retina by the neurodegenerative process. Patients with MS and history of ON, have significantly lower RNFL thickness than do the patients with MS and no history of ON (2). This is also the case of our patient. $\mathrm{He}$ has ON history in the right eye and RNFL is decreased in the temporal sector and, decreased visual acuity. The left eye, with no history for ON, has normal RNFL thickness and normal visual acuity. RNFL thinning is more pronounced in the temporal quadrant in MS patients who have ON history (7).

RNFL thinning reflects axonal loss after an episode of acute $\mathrm{ON}$. This loss will be detected as soon as two months after the acute event. RNFL thinning is stabilized six months after the episode of acute $\mathrm{ON}$, this is why most of the studies are performed after this period of time. Patients with visual impairment after ON have the tendency to adapt to their limitations and will learn to manage with the visual impairment (7). This is most probably the reason why the patient took two years to present to the ophthalmologist.

\section{CONCLUSION}

We presented this case because we want to emphasize the importance of OCT examination in patients with multiple sclerosis and optic neuritis history.

Conflict of interest: none declared Financial support: none declared

\section{REFERENCES}

1. O. Bajenaru. Ghiduri de diagnostic si tratament in neurologie, Ed. Medicala Amaltea, 218-236, 2012

2. Frohman EM. Optical coherence tomography: Aa window into the mechanisms of multiple sclerosis. Nat Clin Pract Neurol. 2008 Dec;4(12): 664-75. Doi: 10.1038/ncpneuro095. 
3. AH Ropper, MA Samuels. Adam's and Victor's Principles of Neurology, $9^{\text {th }}$ Edition, Mc Graw. Hill Medical, pag. 803-805, 2009

4. LP Rowland, TA Padlet. Merritt's Neurology, $12^{\text {th }}$ Edition, Lippincott Williams and Wilkins, 2010

5. Marieta Dumitrache. Explorari si Investigatii in Oftalmologie, Ed. Universitara "Carol Davila", Bucuresti, 2011
6. Maria Satue et al. Optical Coherence Tomography as a Biomarker for Diagnosis, Progression, and Prognosis of Neurodegenerative Diseases. J Ophthalmol. 2016; 2016: 8503859.

7. Susana Noval et al. Optical Coherence Tomography in Multiple Sclerosis and Neuromyelitis Optica: An Update. Multiple Sclerosis International, Volume 2011, Article ID 472790 